\title{
REDUCING THE TIME OF TASK EXECUTION WITH EXISTING RESOURCES - COMPARISON OF APPROACHES
}

\author{
Zupancic, D. ; Buchmeister, B. ${ }^{* *} \&$ Aljaz, T. ${ }^{* * *}$ \\ *Gorenje Household Appliances, Partizanska 12, 3320 Velenje, Slovenia \\ ** University of Maribor, Faculty of Mechanical Engineering, Smetanova 17, 2000 Maribor, Slovenia \\ ${ }^{* * * *}$ Faculty of Information Studies, Ljubljanska 31a, 8000 Novo mesto, Slovenia \\ E-Mail: dusan.zupancic@gorenje.com, borut.buchmeister@um.si; tomaz.aljaz@gmail.com
}

\begin{abstract}
Today, success represents sustainability in introducing new standards and orientation toward the customer, to the quality and price of products, to flexibility, agility and promptness, to economising in resources and protection of the environment. This is not easy to achieve, especially in an environment, where it is natural desire to keep resources busy, in particular the critical resources. This usually results in unfavourable outcomes, usually experiencing significant degradation in performance of company and a lot of firefighting inside the company.

This paper outlines three approaches for manufacturing scheduling, starting with traditional push approach (MRP) and continuing with Kanban (pull) and Theory of Constraints (pull/push), regarding performance and throughput improvement. It determines how resources should be scheduled within a system in order to enhance performance, provide stability and predictability of products. Simulations demonstrate that $200 \%$ to $600 \%$ of improvement is possible at inventory level (work in progress) and the same at lead time, based on the number of completed tasks over a given period of time.

(Received in February 2017, accepted in May 2017. This paper was with the authors 1 month for 1 revision.)
\end{abstract}

Key Words: Scheduling, Kanban, Theory of Constraints, Comparison, Lead Time, WIP

\section{INTRODUCTION}

On the market, which is shaped by increasingly more demanding customers, the rising number of providers and the competitiveness between them, and right business and production strategy are the deciding factors of a company's success $[1,2]$. Companies are searching how to efficiently use their resources. Time is a limiting unrecoverable resource and we must schedule our tasks to utilise this limited resource in an optimum manner. Efficient use of time is namely one of the greatest indicators of competitiveness. In order to achieve that, they are heavily loading system's resources, so that the key resources are always busy. On the other hand, the system is strong as the weakest chain in a system. Therefore, the capacity or throughput of the system is limited by the weakest link, called capacity constrained resources (CCR) [3].

Clearly, having less work in the system will result in starvation of CCR and consequently also in reduced throughput of the system. On the other hand, due dates and quality requirements of customers are one of the major criteria to retain and build competitive advantage on markets. However, this is difficult to achieve, if the CCRs are overloaded with tasks. Therefore, the need for solution is identified that will schedule tasks throughout the system and will limit the number of active tasks and increase predictability and flow stability through it.

Traditional, push based approach is usually suggested for products with small demand uncertainty, as the forecast will provide a good indication of what to produce and keep in inventory, and also for products with high importance of economies of scale in reducing costs. The scheduling functions in a company rely on mathematical techniques and heuristic methods to allocate limited resources to the activities that have to be done. Beside classic 
dispatching rules to sequence the work waiting at workstations plenty of non-conventional methods (algorithms using artificial intelligence) are applied for optimization of scheduling problems [4-9]. Objectives can take many different forms, such as minimizing the time to complete all activities, minimizing the number of activities that are completed after the committed due dates, and so on.

Two advanced general approaches have been widely used in the past decades in manufacturing companies in order to improve throughput of the system, thus reducing number of active tasks in a system: Kanban and Theory of Constraints (TOC) [10].

Kanban [11], translated signboard or billboard in Japanese, is a scheduling system to control number of active tasks in a system, developed by Taiichi Ohno, an industrial engineer at Toyota. Kanban became an effective tool to support running a production system as a whole, and a way to promote improvement. One key indicator of the success of production scheduling based on demand, pushing, is the ability of the demand-forecast to create such a push. Kanban, by contrast, is part of an approach where the "pull" comes from demand. Resupply or production is determined according to the actual demand of the customer. In contexts where supply time is lengthy and demand is difficult to forecast, often, the best one can do is to respond quickly to observed demand. This situation is exactly what a Kanban system accomplishes, in that it is used as a demand signal that immediately travels through the supply chain. Where the supply response is not quick enough to meet actual demand fluctuations, thereby causing potential lost sales, stock building may be deemed more appropriate, and is achieved by placing more kanban in the system.

Second approach used in our paper, was introduced by Eliyahu M. Goldratt, is called Theory of Constraints (TOC) [12]. Despite its name it is not particularly theoretical. Rather, its tools and applications are designed to solve business problems in a practical and effective manner. The methodology assumes that every system or organization can be characterized as network of interdependent processes or elements. This means that systems are analogous to chains, or networks of chains. Like a chain, the system performance is limited by the weakest link - constraint. This means that no matter how much effort you put into improving the processes of a system, only the improvements to the weakest link will produce any detectable system improvement [13-16]. TOC provides tools and applications that enable organizations to identify the constraints (or few of them), exploits them and subordinates others based on that decision in order to get the most out of the existing system or organization. To accomplish this, TOC shifts the focus of management from optimizing separate organizational units, functions and resources to increasing the flow of throughput generated by the entire system. TOC's key processes are focused on removing barriers that prevent each part from working together as an integrated whole. TOC becomes an important problem structuring and solving methodology which changes the way of thinking of managers [17-19].

Additionally, the Drum-Buffer-Rope (DBR) [16, 20, 21] is powerful and robust TOC solution that is intended to manage the flow of work through a (development) process rather than managing the capacity of resources. It is designed to protect against general cause variation that cannot be removed from the system and some special cause variation (e.g., Murphy). As basis for its work it uses first three steps of five focusing steps defined by TOC: identify the system constraint, decide how to exploit the system constraint and subordinate everything else to the above decisions. Basic principle of DBR is shown in Fig. 1.

A lot of researchers have simulated DBR systems, sometimes to estimate DBR's parameters, such as the time buffer, or to compare DBR's effectiveness with other systems. The analysis presented in this paper offers a simple methodology to help decide when to release additional work to the system. A discrete-event simulation model was developed to compare and evaluate traditional manufacturing scheduling approach with Kanban and TOC. The simulator has been written in C\# as a WPF program in Microsoft Visual Studio 2015. 


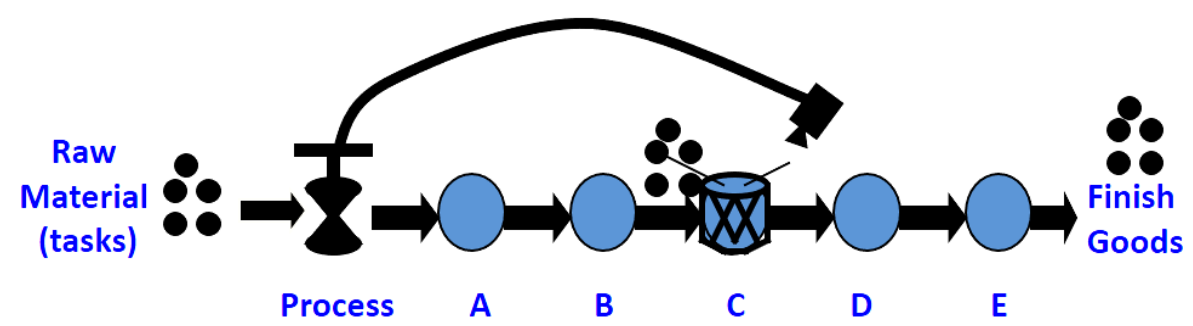

Figure 1: Basic principles of Drum-Buffer-Rope (DBR).

\subsection{Problem definition}

Through the simulation, we want to answer the following questions:

1. What are the average task execution times (lead time) for traditional manufacturing (to be used as a baseline)?

2. How does the average task execution time (lead time) for Kanban compare to the average time for traditional manufacturing at the same process?

3. How does the average task execution time for TOC compare to the average time for traditional manufacturing at the same process?

4. How does the average task execution time for TOC compare to the average time for Kanban at the same process?

5. Which methodology gives the best results considering average task execution time, standard deviation for task execution time, and lowest number of active tasks / inventory (Work-in-Progress - WIP level)?

\section{SIMULATION SETUP}

As it is described in [22] the Dice Game is intended to demonstrate the influence of the variability (or statistical fluctuations as they were named by Goldratt [10]). For simulation, we are using balanced dices (with 4, 6, 8, 12, 16 and 20 surfaces) distributed on workplaces. Each workplace has one operator and one machine, represented by the player and the dice (in each cycle player throws the dice ones and the value of the dice represents the production of that workplace for cycle). Workplaces are ordered in one linear sequence. At the Product Orders we have unlimited supply of tokens and for Finished Products we assume that they are all purchased directly from the end of the production line. Each workplace has a WIP storage, where finished (semi)products from the previous cycle are stored.

Simulation model (for all cases) consists of 6 workplaces (Fig. 2) with balanced dice assigned as follows:

- PLAN - max. 8 products per cycle,

- DM1 - max. 20 semi-finished products per cycle,

- DM2 - max. 16 semi-finished products per cycle,

- DM3 - max. 12 semi-finished products per cycle,

- DM4 - max. 4 semi-finished products per cycle,

- DM5 - max. 6 finished products per cycle.

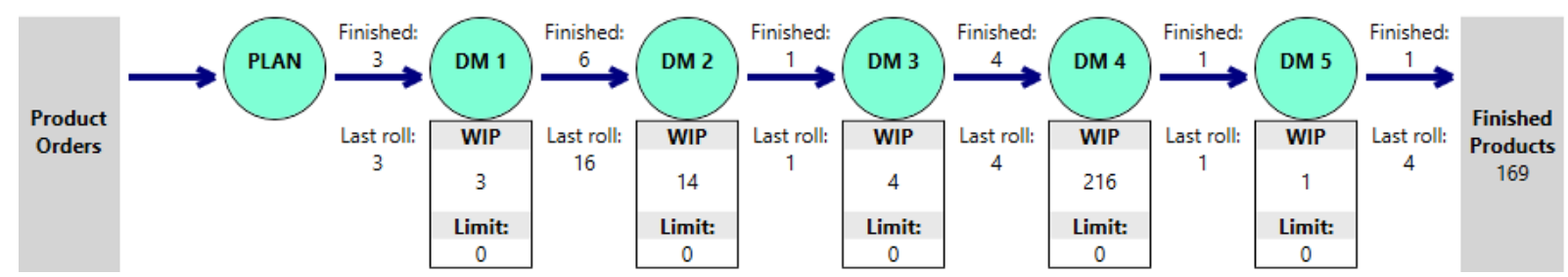

Figure 2: Process setup in simulation (during sample simulation in the simulator). 
Basic rules for the simulation:

- Each workplace has on unbalanced dice with different number of surfaces.

- One cycle is one dice roll at each workplace representing the productivity for one 8-hour shift.

- In each cycle, all workplaces perform the dice roll at the same time (since they are all working in the same 8-hour shift).

- First workplace (PLAN) plays the role of the gatekeeper and pulls the material from unlimited supply.

- All other workplaces pull needed material from the WIP of previous workplace.

○ Each operator can only take the maximum amount available at previous workplace WIP.

o If there is not enough available material in the WIP of previous workplace, the operator can't wait for additional material from current simulation step.

- We start the simulation with zero material in each workplace WIP.

Other assumptions for the simulation:

- We have a simplified process:

$\circ$ No lead time at the beginning of each cycle,

o At each workplace, we only have one machine (if we have more, they are represented with higher available maximum productivity and are considered as one, more productive, machine).

- No problems with workforce and logistics:

○ No prioritizations of tasks,

o Independent process - no artificial delays,

○ All input materials are available on demand,

o No sick leave of workers or mechanical failures on machines,

o Customer buys all finished products immediately.

\subsection{Simulation setup for traditional production scheduling}

Simulation setup for traditional scheduling is set up with no constrains on WIP with maximum throughput based on dice roll in each cycle.

Each simulation will run for 100 cycles with 20 replications.

\subsection{Simulation setup for kanban production}

Simulation setup for kanban production is set up with the constraint of 5 semi-finished products in WIP for each workplace (estimation based on trial runs).

Each simulation will run for 100 cycles with 20 replications.

\subsection{Simulation setup for DBR (TOC) production}

Based on results of simulation for traditional production scheduling, it is established, that CCR (Capacity Constrained Resource) is workplace DM4 and it plays the role of the Drum in simulation of this process. Buffer capacity is set to 15 semi-finished products based on trial runs.

Each simulation will run for 100 cycles with 20 replications.

\section{RESULTS AND DISCUSSION}

In Table I the following parameters are displayed on the horizontal axis for each process, based on 20 simulations:

- Min. - minimal value among 20 performed simulations,

- Max. - maximum value among 20 performed simulations, 
- Avg. - average value among 20 performed simulations.

On vertical axis, the following parameter values are displayed:

- End WIP - WIP after the end of simulation,

- Finished products - quantity of finished products after 100 cycles of simulation,

- Production time, min. - minimum production time in 100 cycles of simulation,

- Production time, max. - maximum production time in 100 cycles of simulation,

- Production time, avg. - average production time for 100 cycles of simulation,

- Standard deviation - standard deviation of production time for 100 cycles of simulation.

Table I: Summary of 60 simulations.

\begin{tabular}{|l|c|c|c|c|c|c|c|c|c|}
\cline { 2 - 11 } \multicolumn{1}{c|}{ Approach: } & \multicolumn{4}{|c|}{ Traditional } & \multicolumn{4}{c|}{ Kanban } & \multicolumn{3}{c|}{ DBR } \\
\cline { 2 - 11 } \multicolumn{1}{c|}{} & Min. & Max. & Avg. & Min. & Max. & Avg. & Min. & Max. & Avg. \\
\hline End WIP & 184 & 265 & $\mathbf{2 1 7 , 5 5}$ & 12 & 17 & $\mathbf{1 4}$ & 14 & 18 & $\mathbf{1 5 , 3 5}$ \\
\hline Finished products & 173 & 203 & $\mathbf{1 8 6 , 3 5}$ & 161 & 181 & $\mathbf{1 7 2 , 1}$ & 172 & 205 & $\mathbf{1 8 5 , 2 5}$ \\
\hline Production time, min. & 5 & 5 & $\mathbf{5}$ & 5 & 5 & $\mathbf{5}$ & 5 & 5 & $\mathbf{5}$ \\
\hline Production time, max. & 46 & 62 & $\mathbf{5 3 , 2}$ & 9 & 12 & $\mathbf{1 0 , 4 5}$ & 9 & 15 & $\mathbf{1 1 , 1 5}$ \\
\hline Production time, avg. & 25 & 35 & $\mathbf{2 9 , 1}$ & 7 & 8 & $\mathbf{7 , 2}$ & 6 & 8 & $\mathbf{7 , 0 5}$ \\
\hline Standard deviation & 11,77 & 17,88 & $\mathbf{1 3 , 9 2 7}$ & 0,76 & 1,54 & $\mathbf{1 , 1 7 2}$ & 0,91 & 1,71 & $\mathbf{1 , 2 2 8 5}$ \\
\hline
\end{tabular}

\subsection{Results by used approach}

- Traditional production scheduling

Simulation of Traditional production scheduling serves as a baseline for comparison with other approaches, for initial WIP restriction and for CCR identification.

Table II: Traditional production scheduling simulations summary.

\begin{tabular}{|c|c|c|c|c|c|c|}
\hline & & & \multicolumn{4}{|c|}{ Duration } \\
\cline { 4 - 7 } Iteration & End WIP & Finished & Min. & Max. & Avg. & Std. dev. \\
\hline 1 & 205 & 176 & 5 & 50 & 30 & 13,36 \\
\hline 2 & 240 & 185 & 5 & 54 & 29 & 14,13 \\
\hline 3 & 192 & 195 & 5 & 48 & 27 & 12,73 \\
\hline 4 & 214 & 183 & 5 & 52 & 30 & 14,11 \\
\hline 5 & 248 & 186 & 5 & 60 & 35 & 14,19 \\
\hline 6 & 244 & 173 & 5 & 62 & 31 & 17,88 \\
\hline $\mathbf{7}$ & $\mathbf{2 1 9}$ & $\mathbf{1 9 3}$ & $\mathbf{5}$ & $\mathbf{5 2}$ & $\mathbf{3 1}$ & $\mathbf{1 3 , 9 9}$ \\
\hline 8 & 195 & 181 & 5 & 55 & 31 & 13,54 \\
\hline 9 & 220 & 189 & 5 & 53 & 27 & 14,34 \\
\hline 10 & 210 & 194 & 5 & 50 & 27 & 13,28 \\
\hline 11 & 184 & 181 & 5 & 47 & 25 & 12,64 \\
\hline 12 & 188 & 186 & 5 & 52 & 29 & 12,46 \\
\hline 13 & 189 & 187 & 5 & 46 & 28 & 11,77 \\
\hline 14 & 199 & 200 & 5 & 50 & 26 & 13,26 \\
\hline 15 & 220 & 186 & 5 & 56 & 32 & 15,87 \\
\hline 16 & 249 & 194 & 5 & 56 & 29 & 14,48 \\
\hline 17 & 265 & 173 & 5 & 56 & 30 & 14,13 \\
\hline 18 & 218 & 180 & 5 & 57 & 29 & 14,56 \\
\hline 19 & 255 & 182 & 5 & 57 & 29 & 14,77 \\
\hline 20 & 197 & 203 & 5 & 51 & 27 & 13,05 \\
\hline
\end{tabular}




\begin{tabular}{|c|c|c|c|c|c|c|}
\hline Min. & 184 & 173 & 5 & 46 & 25 & 11,77 \\
\hline Max. & 265 & 203 & 5 & 62 & 35 & 17,88 \\
\hline Avg. & 217,55 & 186,35 & 5 & 53,2 & 29,1 & 13,927 \\
\hline
\end{tabular}

Table II shows that average number of finished products in 100 iterations was 186,35. End WIP after 100 iterations is 217,55 , which represents $177 \%$ of finished products.

Fastest production time was 5 cycles while slowest was on average 53,2 cycles resulting in production time average of 29,1 cycle with average standard deviation of 13,927 cycles.

Iteration no. 7 is closest to these averages and is used for further more detailed analysis.

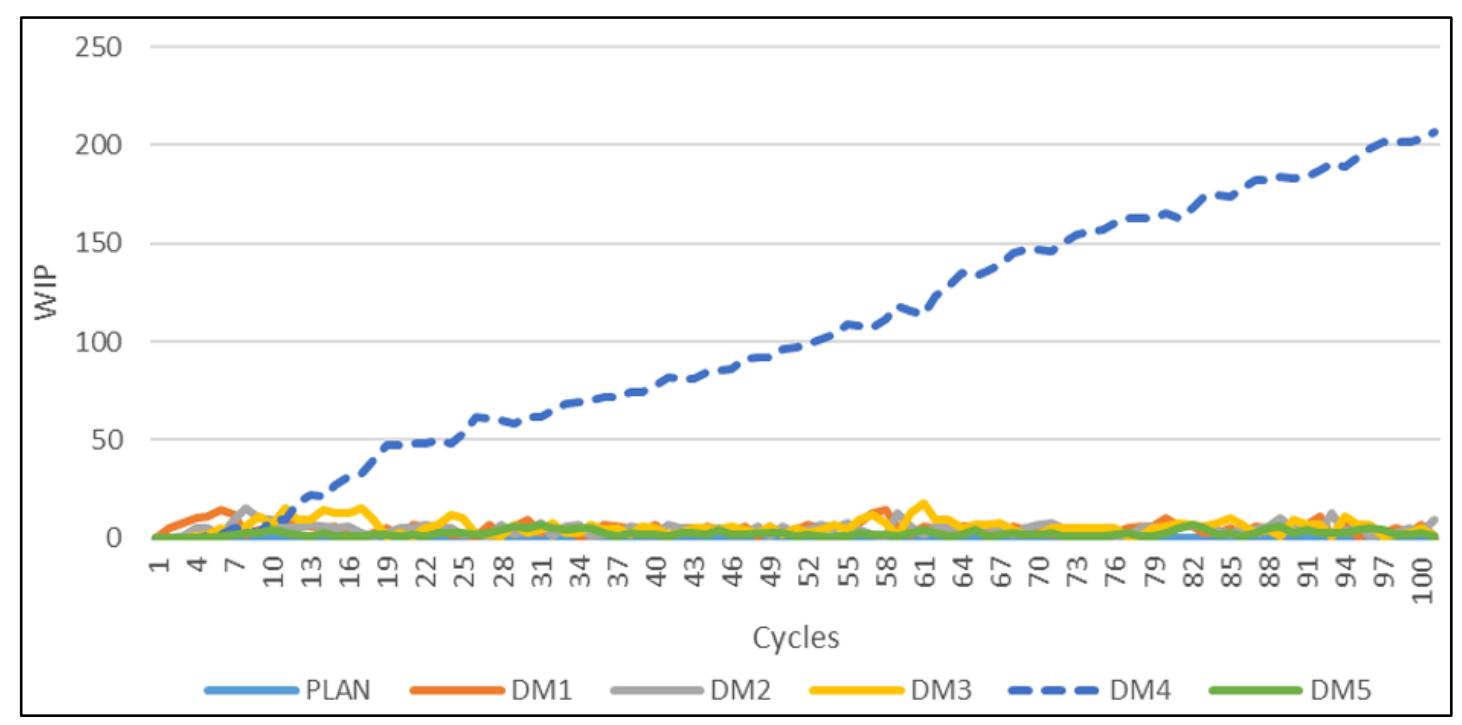

Figure 3: Simulation 7 - current cycle WIP at workplaces.

Fig. 3 reveals that WIP is relatively stable around 5 on all workplaces, except before workplace DM4, where they linearly grow. This means, that our CCR is workplace DM4.

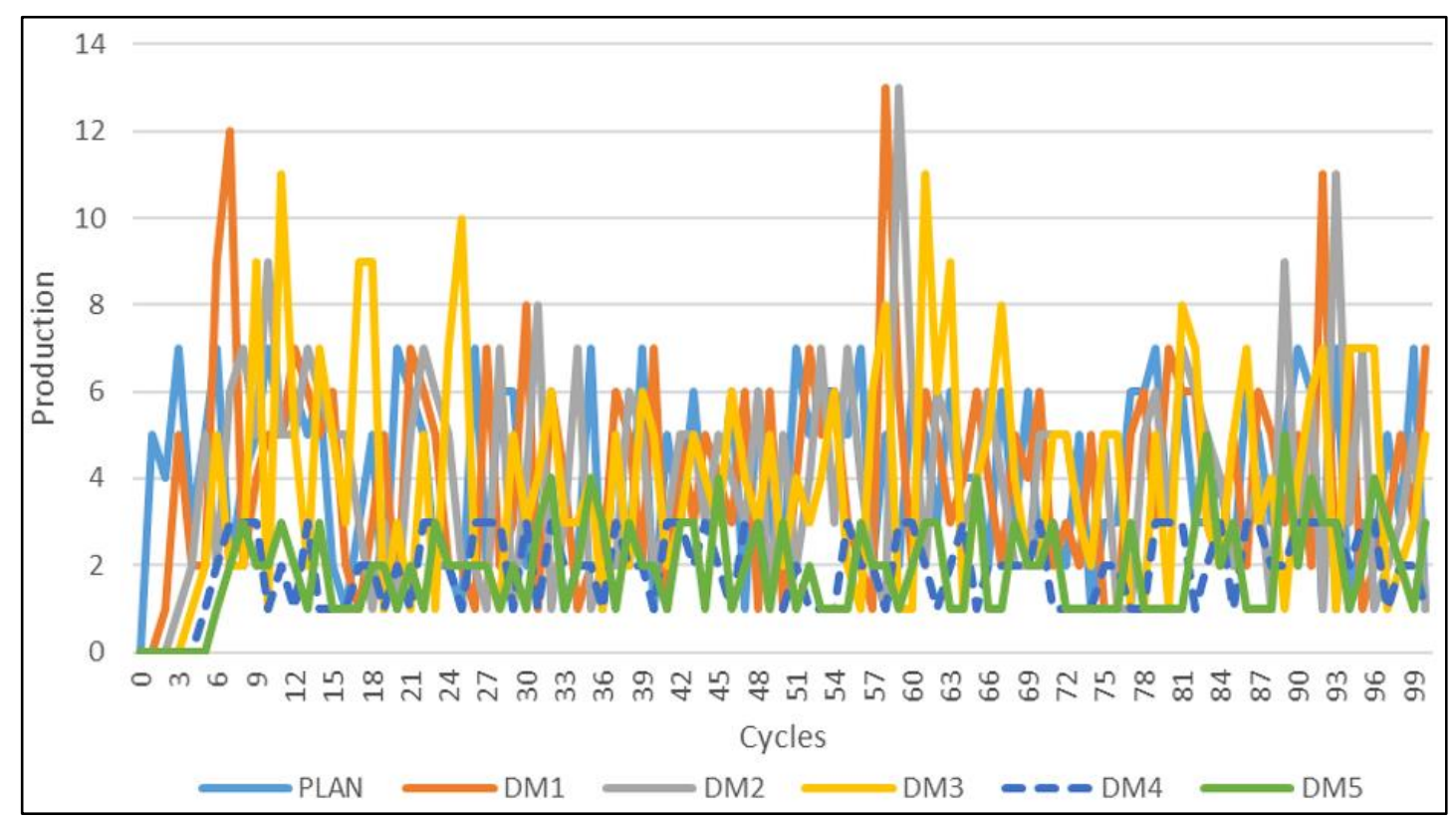

Figure 4: Simulation 7 - production at workplaces.

Fig. 4 displays production (flow) for each workplace by iterations. There are no production outages (due to lack of material at previous workplace WIP) detected except at the 
beginning of production (due to lead time). Chart also reveals that DM5 is not working at full capacity because of the limited supply from the DM4. Production on other workplaces is within expected statistical variance.

- Kanban

Based on the results for Traditional production scheduling, where WIP was around 5 on almost all workplaces except CCR, this number was used as WIP constraint for each workplace WIP.

Table III: Kanban simulations summary.

\begin{tabular}{|c|c|c|c|c|c|c|}
\hline & & & \multicolumn{4}{|c|}{ Duration } \\
\cline { 4 - 7 } Iteration & WIP & Finished & Min. & Max. & Avg. & Std. dev. \\
\hline 1 & 13 & 173 & 5 & 11 & 7 & 1,53 \\
\hline 2 & 16 & 172 & 5 & 10 & 7 & 1 \\
\hline 3 & 14 & 166 & 5 & 10 & 8 & 0,99 \\
\hline 4 & 13 & 176 & 5 & 10 & 7 & 1,25 \\
\hline 5 & 12 & 173 & 5 & 11 & 7 & 1,28 \\
\hline 6 & 14 & 171 & 5 & 11 & 7 & 1,08 \\
\hline 7 & 15 & 177 & 5 & 9 & 7 & 0,76 \\
\hline 8 & 15 & 173 & 5 & 10 & 7 & 0,98 \\
\hline 9 & 12 & 177 & 5 & 9 & 7 & 1,03 \\
\hline 10 & 17 & 165 & 5 & 11 & 7 & 1,4 \\
\hline 11 & 13 & 177 & 5 & 10 & 7 & 1,03 \\
\hline 12 & 14 & 181 & 5 & 10 & 7 & 0,9 \\
\hline 13 & 16 & 171 & 5 & 11 & 7 & 1,32 \\
\hline 14 & 14 & 177 & 5 & 10 & 7 & 1,22 \\
\hline 15 & 13 & 166 & 5 & 10 & 8 & 1,14 \\
\hline $\mathbf{1 6}$ & $\mathbf{1 4}$ & $\mathbf{1 7 2}$ & $\mathbf{5}$ & $\mathbf{1 0}$ & $\mathbf{7}$ & $\mathbf{1 , 2 1}$ \\
\hline 17 & 13 & 176 & 5 & 11 & 7 & 1,16 \\
\hline 18 & 12 & 176 & 5 & 11 & 7 & 1,54 \\
\hline 19 & 15 & 161 & 5 & 12 & 8 & 1,47 \\
\hline 20 & 15 & 162 & 5 & 12 & 8 & 1,15 \\
\hline Min. & 12 & 161 & 5 & 9 & 7 & 0,76 \\
\hline Max. & 17 & 181 & 5 & 12 & 8 & 1,54 \\
\hline Avg. & 14 & 172,1 & 5 & 10,45 & 7,2 & 1,172 \\
\hline
\end{tabular}

Table III shows that the average number of finished products in 100 iterations was 172,1 (which represents $92 \%$ of production with Traditional production scheduling). Average end WIP after 100 iterations is 14, which represents $8 \%$ of finished products. End WIP with Kanban represents only $3 \%$ of end WIP with Traditional production scheduling.

Fastest production time was 5 cycles while slowest was on average 10,45 cycles resulting in production time average of 7,2 cycle with average standard deviation of 1,127 cycles.

Iteration no. 16 is closest to these averages and is used for further more detailed analysis.

Fig. 5 reveals that WIP is relatively stable at all workplaces, except at workplace DM5, where they are the lowest due to limited supply from DM4 which was already identified as CCR.

Fig. 6 displays production (flow) for each workplace by iterations. There are no production outages (due to lack of material at previous workplace WIP) detected except at the beginning of production (due to lead time). Chart also reveals that production at all workplaces is relatively synchronized. 


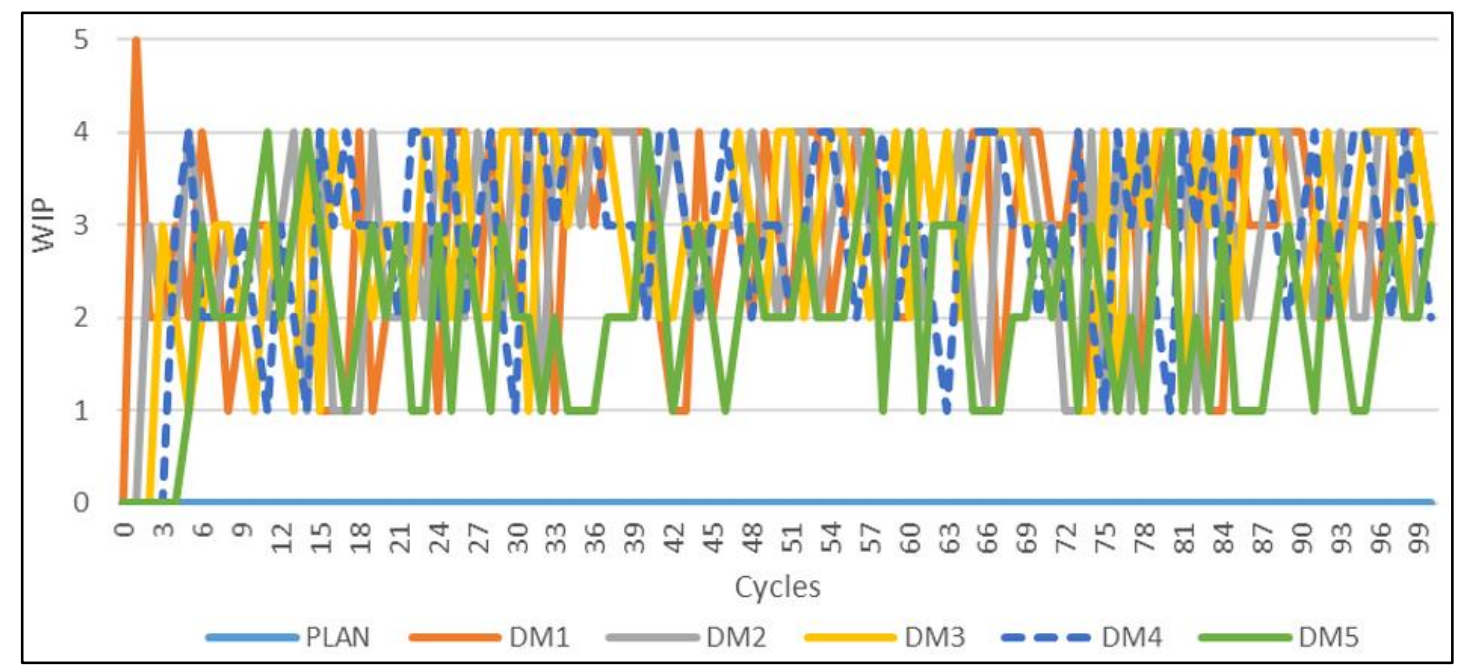

Figure 5: Simulation 16 - current cycle WIP at workplaces.

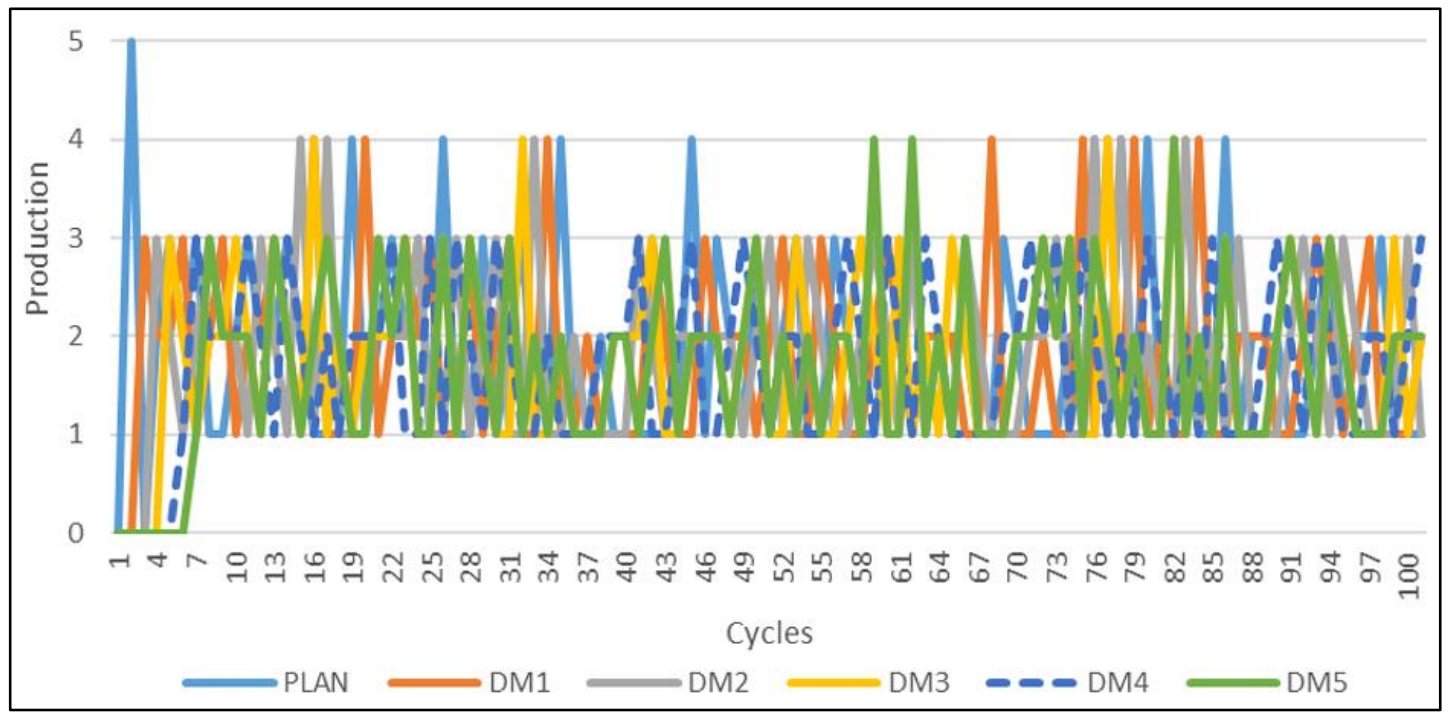

Figure 6: Simulation 16 - production at workplaces.

- $\mathrm{TOC}-\mathrm{DBR}$

Based on results for Traditional production scheduling, where WIP at all workplaces before CCR was around 5, this value was used for buffer size estimation. Since there are three workplaces before CCR/DM4 (DM1, DM2 and DM3 - PLAN is in our process only gatekeeper), size of the buffer was set to 15 .

Table IV shows that average number of finished products in 100 iterations was 185,25 (which represents $99 \%$ of production with Traditional production scheduling). Average end WIP after 100 iterations is 15,35 , which represents $8,2 \%$ of finished products.

Fastest production time was 5 cycles while slowest was on average 11,15 cycles resulting in production time average of 7,05 cycle with average standard deviation of 1,2285 cycles.

Iteration no. 18 is closest to this averages and is used for further more detailed analysis.

Fig. 7 reveals that WIP is relatively stable at all workplaces except WIP before DM4 (already identified as CCR) which varies between 3 and 10.

Fig. 8 displays production (flow) for each workplace by iterations. There are no production outages (due to lack of material at previous workplace WIP) detected, except on the beginning of production (due to lead time). Chart also reveals that production at all workplaces is synchronized resulting in smooth production flow. 
Table IV: DBR simulations summary.

\begin{tabular}{|c|c|c|c|c|c|c|}
\hline & & & \multicolumn{5}{|c|}{ Duration } \\
\cline { 4 - 7 } Iteration & WIP & Finished & Min. & Max. & Avg. & Std. dev. \\
\hline 1 & 15 & 175 & 5 & 11 & 8 & 1,31 \\
\hline 2 & 16 & 178 & 5 & 11 & 7 & 1,44 \\
\hline 3 & 15 & 194 & 5 & 10 & 7 & 1,05 \\
\hline 4 & 15 & 179 & 5 & 12 & 7 & 1,14 \\
\hline 5 & 15 & 199 & 5 & 11 & 7 & 0,99 \\
\hline 6 & 15 & 172 & 5 & 15 & 8 & 1,21 \\
\hline 7 & 15 & 181 & 5 & 11 & 6 & 1,21 \\
\hline 8 & 17 & 189 & 5 & 10 & 7 & 1 \\
\hline 9 & 14 & 189 & 5 & 11 & 7 & 1,26 \\
\hline 10 & 15 & 184 & 5 & 12 & 7 & 1,28 \\
\hline 11 & 16 & 183 & 5 & 13 & 7 & 1,67 \\
\hline 12 & 16 & 187 & 5 & 11 & 7 & 1,14 \\
\hline 13 & 16 & 185 & 5 & 11 & 7 & 1,37 \\
\hline 14 & 14 & 205 & 5 & 9 & 6 & 0,91 \\
\hline 15 & 15 & 174 & 5 & 11 & 8 & 1,23 \\
\hline 16 & 18 & 177 & 5 & 12 & 7 & 1,71 \\
\hline 17 & 15 & 188 & 5 & 10 & 7 & 0,93 \\
\hline $\mathbf{1 8}$ & $\mathbf{1 6}$ & $\mathbf{1 8 4}$ & $\mathbf{5}$ & $\mathbf{1 1}$ & $\mathbf{7}$ & $\mathbf{1 , 2 7}$ \\
\hline 19 & 14 & 197 & 5 & 10 & 7 & 1,15 \\
\hline 20 & 15 & 185 & 5 & 11 & 7 & 1,3 \\
\hline Min. & 14 & 172 & 5 & 9 & 6 & 0,91 \\
\hline Max. & 18 & 205 & 5 & 15 & 8 & 1,71 \\
\hline Avg. & 15,35 & 185,25 & 5 & 11,15 & 7,05 & 1,2285 \\
\hline & & & & & & \\
\hline
\end{tabular}

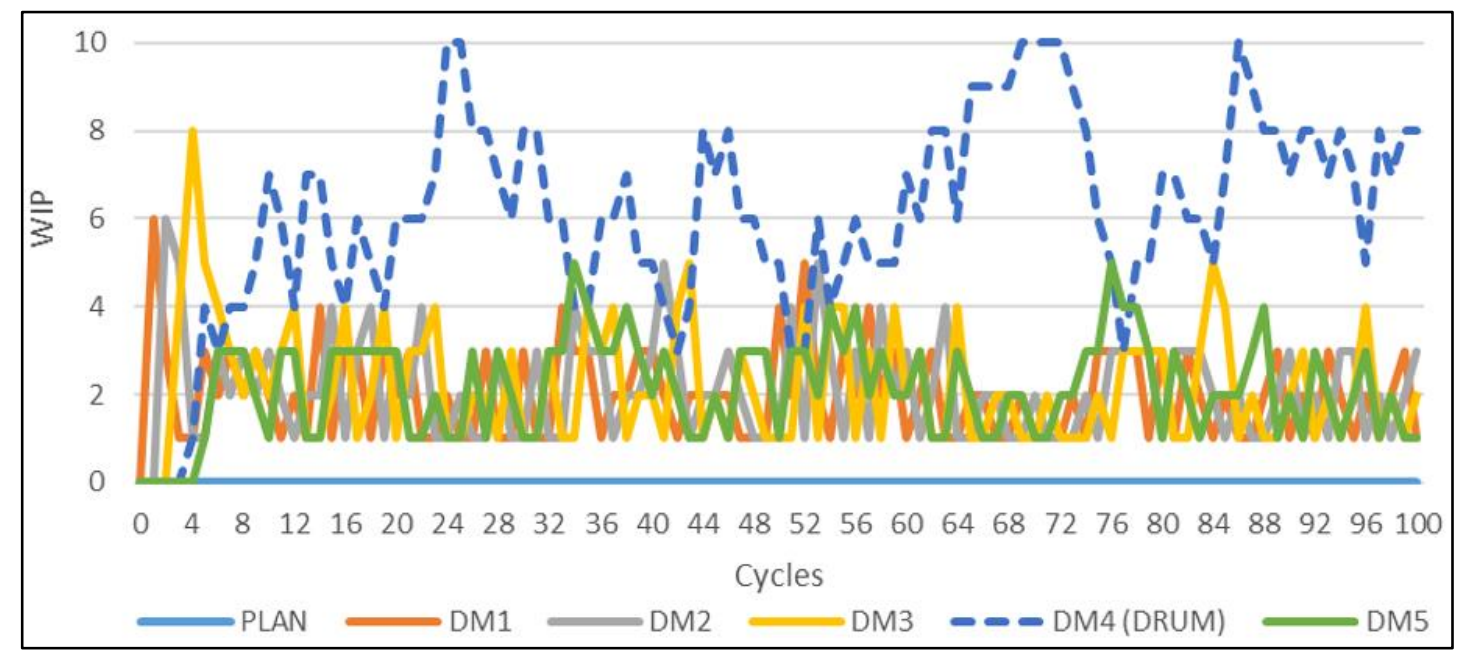

Figure 7: Simulation 18 - WIP before each workplace. 


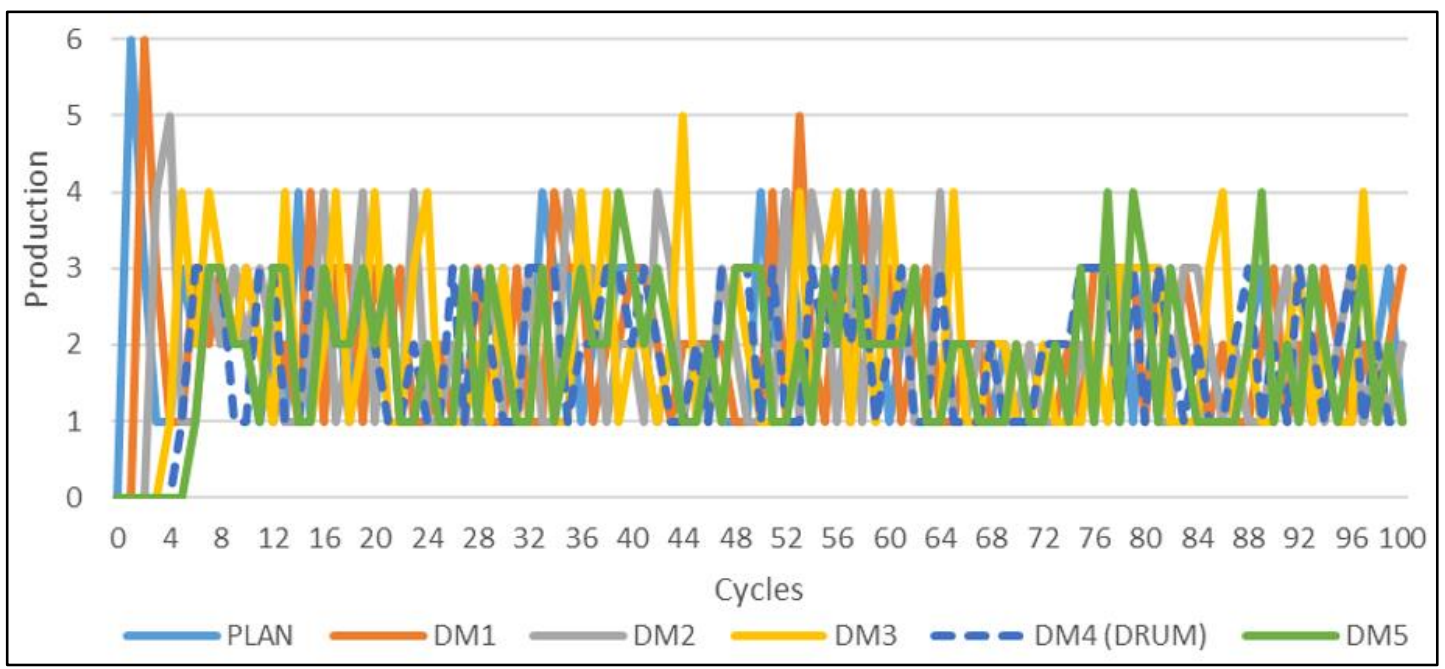

Figure 8: Simulation 18 - production at workplaces.

\subsection{Comparative analysis by used approach}

- WIP

Since WIP represents the cost in our process and at the same time it acts as a buffer, it is ideally low, but not too low to cause delays in the manufacturing process.

Fig. 9 displays the area of End WIP values for 20 simulations for each process type. It is evident that End WIP is highest for Traditional production scheduling and that this approach has the highest dispersion of results among all three approaches (ranging from 184 to 256).

End WIP for Kanban and DBR is considerably lower and of similar size (Kanban: from 12 to 17; DBR: from 14 to 18 ) with lower dispersion of results.

Results show that from End WIP size management point Kanban and DBR give better result.

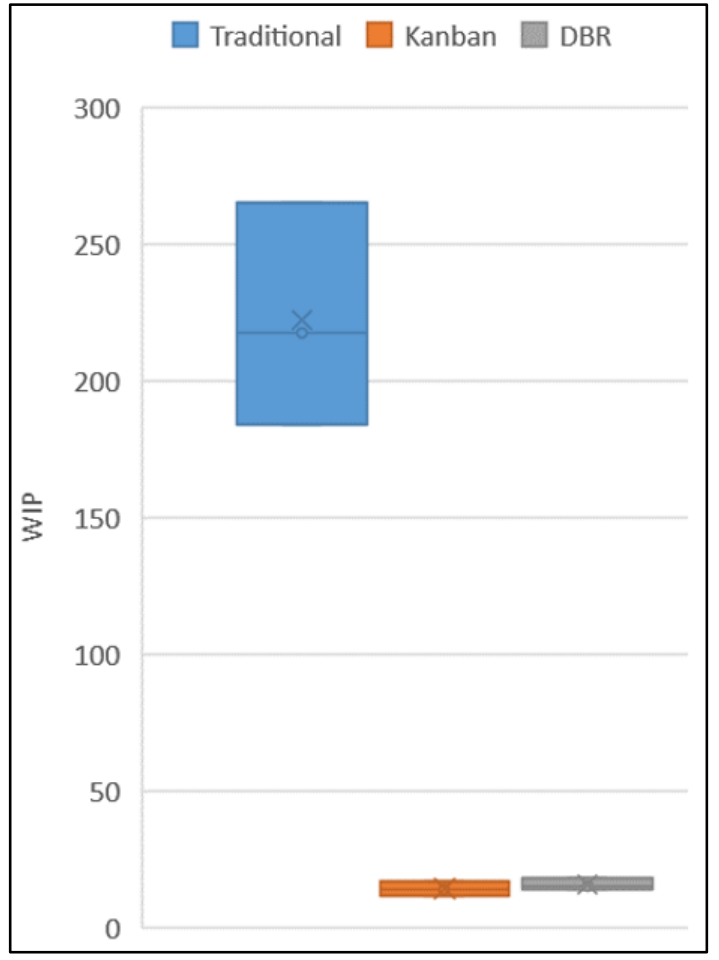

Figure 9: Quartile chart of End WIP simulation results by used approach.

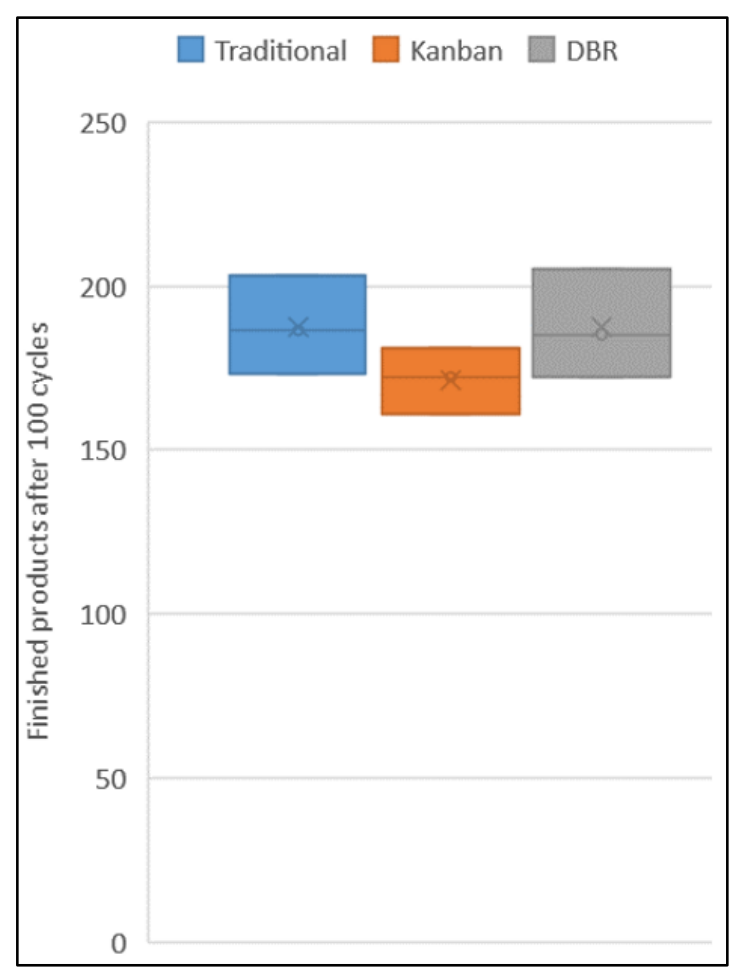

Figure 10: Quartile chart of Finished products simulation results by used approach. 
- Finished products

Number of finished products provides insight into process throughput and higher values are more desirable.

Fig. 10 displays the area of values for finished products where we can see a similar number and dispersion of finished products values for Traditional production scheduling (from 173 to 203) and DBR (from 172 to 205).

Kanban is slightly lower in this category (from 161 to 181) but has smaller dispersion of results.

- Average production time

Production time is important for product delivery planning, so lower values are more desirable. Moreover, we also want low standard deviation of production times, so we can plan product delivery time with higher confidence.

Fig. 11 displays the area of values for average production times. It is clearly noticeable that these values are highest for Traditional production scheduling (from 25 to 35 cycles) with highest dispersion. Results for Kanban (from 7 to 8 cycles) and DBR (6 to 8 cycles) are comparable and considerably lower (slightly higher than theoretical minimum of 5 cycles) with low dispersion.

Let's look at the second criteria connected to previous parameter: standard deviation of production times.

Fig. 12 displays the area of values for standard deviations of production times by process type. It is evident that predicting production time is hardest for Traditional production scheduling where standard deviation is from 11,77 to 17,88 cycle with average of 13,927 cycle. Kanban and DBR have comparable results as well. Kanban has standard deviation ranging from 0,76 to 1,54 with average of 1,172 cycle. DBR has standard deviation from 0,91 to 1,71 cycle, with average of 1,2285 cycle.

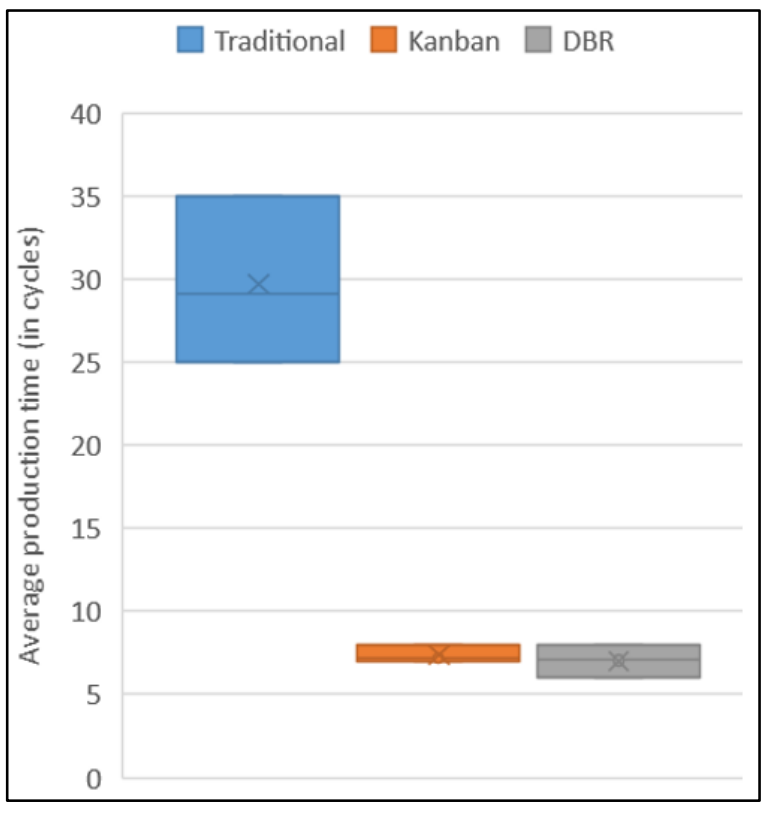

Figure 11: Quartile chart of average production time by used approach.

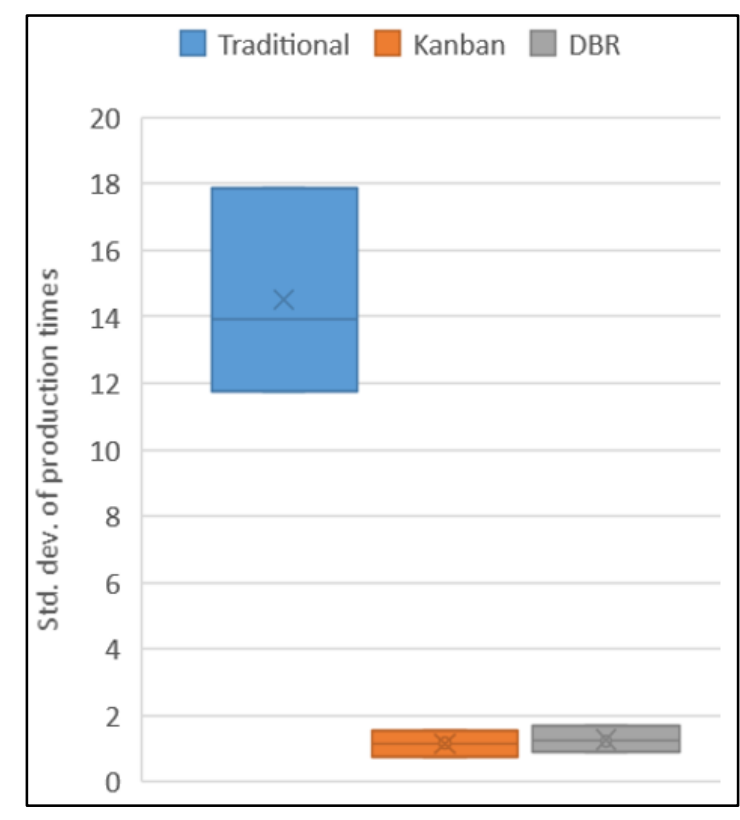

Figure 12: Quartile chart of standard deviation of production times.

With combination of both values the following production times are predicted:

- Traditional production scheduling has average production time of 29,1 cycle and average standard deviation of 13,927 cycle, so we can expect production times from 15 to 43 cycles. 
- Kanban has average production time of 7,2 cycle and average standard deviation of 1,172 cycle, so we can expect production times from 6 to 8 cycles.

- DBR has average production time of 7,05 cycles and average standard deviation of 1,2285 cycle, so we can expect production times from 6 to 8 cycles.

\section{CONCLUSION}

Scheduling as a research area is motivated by questions that arise in production planning and, generally, in all situations in which scarce resources have to be allocated to activities over time. Many manufacturing companies are now critically evaluating their processes to determine their effectiveness in bringing maximum value to customers. They strive to include methods that greatly minimize lead time, reduce costs, and improve quality.

In this paper it has been analysed and formalized some findings using simulator for implementation of Kanban and Theory of Constraints tools on sample manufacturing line. Analyses showed that resource productivity is at its highest with traditional production scheduling, but on the account of high inventory (number of active tasks) at the Capacity Constrained Resource (CCR) and by far the longest time for task completion (lead time). Kanban as well as TOC showed a stable and relatively low number of active tasks and shorter task completion time compared to traditional production scheduling, but on the account of lower resource efficiency.

The key finding of this article is that control over the number of active tasks in a system is more important than continuous full utilization of all resources (efficiency) and significantly impacts production lead time through lower number of active tasks (effectiveness). Additionally, it was showed that introduction of control of active tasks is simpler with Kanban, but TOC offers better results at system fluidity through advanced management of a buffer, and at the same time it leaves much space for improvement in the future with the introduction of Fever Chart for prioritization of tasks that are already within a system.

\section{REFERENCES}

[1] Bagheri, F.; Noorossana, R. (2016). A statistical model for determination of the type of knowledge management approach based on organization processes, Transactions of FAMENA, Vol. 40, No. 3, 43-56, doi:10.21278/TOF.40304

[2] Kapor, N.; Milinovic, M.; Jeremic, O.; Petrovic, D. (2015). Deterministic mathematical modelling of platform performance degradation in cyclic operation regimes, Strojniski vestnik Journal of Mechanical Engineering, Vol. 61, No. 3, 167-175, doi:10.5545/sv-jme.2014.2294

[3] Goldratt, E. M. (2003). Production the TOC Way with Simulator, The North River Press Publishing Corporation, Great Barrington

[4] Tasič, T.; Buchmeister, B.; Ačko, B. (2007). The development of advanced methods for scheduling production processes, Strojniski vestnik - Journal of Mechanical Engineering, Vol. 53, No. 12, 844-857

[5] Rathinam, B.; Govindan, K.; Neelakandan, B.; Raghavan, S. S. (2015). Rule based heuristic approach for minimizing total flow time in permutation flow shop scheduling, Technical Gazette, Vol. 22, No. 1, 25-32, doi:10.17559/TV-20130704132725

[6] Buchmeister, B.; Friscic, D.; Palcic, I. (2013). Impact of demand changes and supply chain's level constraints on bullwhip effect, Advances in Production Engineering \& Management, Vol. 8, No. 4, 199-208, doi:10.14743/apem2013.4.167

[7] Majazi Dalfard, V.; Ranjbar, V. (2012). Multi-projects scheduling with resource constraints \& priority rules by the use of simulated annealing algorithm, Technical Gazette, Vol. 19, No. 3, 493-499 
[8] Tang, M.; Gong, D.; Liu, S.; Zhang, H. (2016). Applying multi-phase particle swarm optimization to solve bulk cargo port scheduling problem, Advances in Production Engineering \& Management, Vol. 11, No. 4, 299-310, doi:10.14743/apem2016.4.228

[9] Li, Y.; Yao, X.; Zhou, J. (2016). Multi-objective optimization of cloud manufacturing service composition with cloud-entropy enhanced genetic algorithm, Strojniski vestnik - Journal of Mechanical Engineering, Vol. 62, No. 10, 577-590, doi:10.5545/sv-jme.2016.3545

[10] Goldratt, E. M.; Cox, J. (2004). The Goal: A Process of Ongoing Improvement, North River Press, Great Barrington

[11] Lu, D. J. (1989). Kanban Just-in Time at Toyota: Management Begins at the Workplace (Volume 1), Productivity Press, Portland

[12] Goldratt, E. M. (1999). Theory of Constraints, North River Press Publishing Corporation, Great Barrington

[13] Simsit, Z. T.; Gunay, N. S.; Vayvay, O. (2014). Theory of constraints: a literature review, Proceedings of the $10^{\text {th }}$ International Strategic Management Conference, 930-936

[14] Naor, M.; Bernardes, E. S.; Coman, A. (2013). Theory of constraints: is it a theory and a good one?, International Journal of Production Research, Vol. 51, No. 2, 542-554, doi: $10.1080 / 00207543.2011 .654137$

[15] Golmohammadi, D. (2015). A study of scheduling under the theory of constraints, International Journal of Production Economics, Vol. 165, 38-50, doi:10.1016/j.ijpe.2015.03.015

[16] Zhang, X. M.; Du, Y. L. (2015). Research of production scheduling based on theory of constraints, Proceedings of the 2015 International Conference on Electrical, Automation and Mechanical Engineering, 142-145

[17] Banerjee, A.; Mukhopadhyay, S. K. (2016). A contemporary TOC innovative thinking process in the backdrop of leagile supply chain, Journal of Enterprise Information Management, Vol. 29, No. 3, 400-431, doi:10.1108/JEIM-08-2014-0086

[18] Golmohammadi, D.; Mansouri, S. A. (2015). Complexity and workload considerations in product mix decisions under the theory of constraints, Naval Research Logistics, Vol. 62, No. 5, 357-369, doi:10.1002/nav.21632

[19] Wang, J.-Q.; Zhang, Z.-T.; Chen, J.; Guo, Y.-Z.; Wang, S.; Sun, S.-D.; Qu, T.; Huang, G. Q. (2014). The TOC-based algorithm for solving multiple constraint resources: a re-examination, IEEE Transactions on Engineering Management, Vol. 61, No. 1, 138-146, doi:10.1109/TEM.2013.2264830

[20] Aljaž, T. (2014). Out of chaos in 12 months - improving lead time of sprint projects in software development implementing Drum Buffer Rope Solution, ERK'2014 Conference Proceedings, 4851

[21] Chakravorty, S. S.; Hales, D. N. (2016). Improving labour relations performance using a Simplified Drum Buffer Rope (S-DBR) technique, Production Planning \& Control, Vol. 27, No. 2, 102-113, doi:10.1080/09537287.2015.1079744

[22] Schragenheim, E.; Dettmer, H. W. (2001). Manufacturing at Warp Speed: Optimizing Supply Chain Financial Performance, CRC Press, Boca Raton 